\title{
Investigation of the Effect of the Front Angle on the Strength of the Cutting Wedge by Photomecanics
}

\author{
Olga Zhed ${ }^{1, *}$, Mikhail Kozochkin ${ }^{2}$, and Katerina Kazymaeva ${ }^{1}$ \\ ${ }^{1}$ Peoples Friendship University of Russia (RUDN University), 6 Miklukho-Maklaya St, Moscow, \\ 117198, Russian Federation \\ ${ }^{2}$ Moscow State University of Technology "STANKIN" (MSUT "STANKIN"), 1 Vadkovsky \\ side-str, Moscow, 127055, Russian Federation
}

\begin{abstract}
Stress-strain state of cutting wedge models is evaluated by photomechanics method in the work. Models are made of optically sensitive organic glass of ED-6 grade. The aim of the study is to improve the method of selecting geometric parameters of the cutting wedge based on experimental data from photomechanics. The effect of the face angle on the strength of the cutting wedge was considered.
\end{abstract}

\section{Introduction}

Polarization-optical method (photomechanics method) of stress analysis is used to simulate the stress state of structures and constructions [1]. The photomechanics method complements analytical calculation methods, which are characterized by the high complexity of setting boundary conditions and taking into account all load factors. The main purpose of the experiments was to study the strength of the cutting wedge from the value of the face angle. The work was carried out in the photomechanics laboratory of the Academy of Engineering RUDN University.

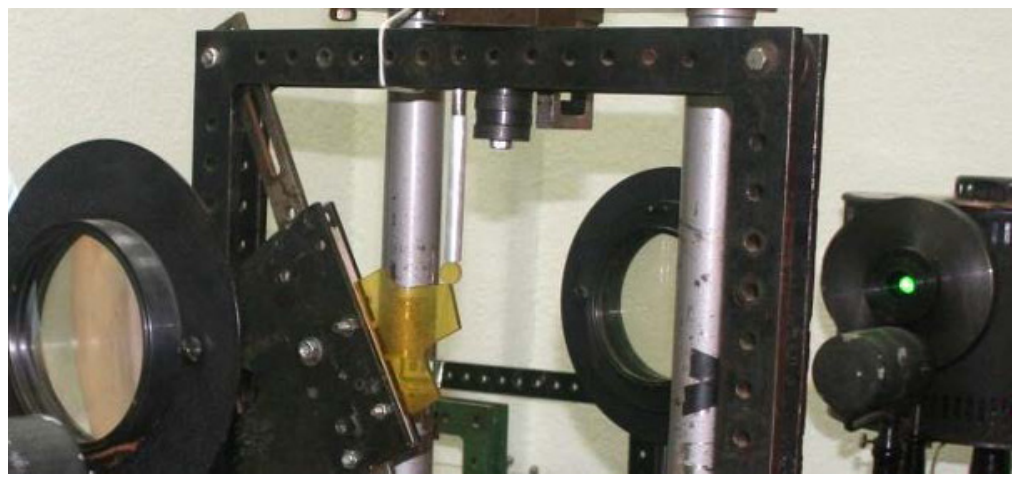

Fig. 1. Experimental stand at the plant PPU-7

\footnotetext{
*Corresponding author: zhed-ov@rudn.ru
} 


\section{Material of the model and technique of physical experiments}

The polarization-optical method [1] allows to obtain important information of the stressstrain state nature, representing areas of tensile and compressive stresses. The image of the interference bands obtained during the study gives a complete idea of the stress field in the transparent model, which corresponds to the specific conditions of the experiment.

As objects of study, two models of a cutting wedge with different face angles were made: negative $\gamma=-15^{\circ}$ and positive $\gamma=+15^{\circ}$. The clearance angle was assumed to be the same $\alpha=10^{\circ}$ for all models. The models were made of an optically sensitive material based on epoxy resin ED-6 MTGFA, which is elastic in the load range under investigation. The experiment procedure involved four consecutive stages of work.

The first stage: calibration. Calibration of the optical properties of the material of which the cutting wedge models were made was carried out by loading a flat disk of the same material. Based on experimental data obtained during calibration, optical constant is calculated from expression [1]:

$$
\sigma_{0}^{1,0}=\left(8 P_{i}\right) /\left(\pi \cdot d \cdot n_{c}\right),
$$

where $\sigma_{0}^{1,0}$ - optical constant, in MPa $\cdot \mathrm{cm} / \mathrm{strip}$ or in $\mathrm{kgf} / \mathrm{cm} / \mathrm{strip} ; P_{i}-$ the load applied to the disk during calibration, in $\mathrm{H}$ or in $\mathrm{kgf} ; n_{c}$ is the number of interference strips in the center of the disk with a diameter of $d=1.0 \mathrm{~cm}$. The optical constant of the material of the cutting wedge models was $\sigma_{0}^{1,0}=19.1 \mathrm{kgf} / \mathrm{cm} / \mathrm{strip}$.

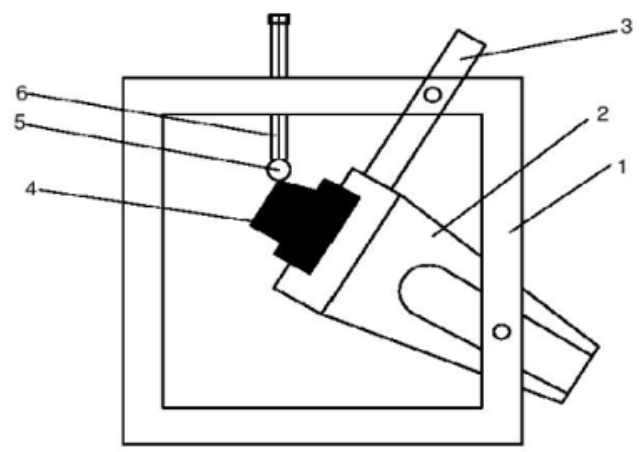

Fig. 2. Model loading diagram: 1 - frame, 2 - plate, 3 - thrust, 4 - cutting wedge model, 5 - calibration disk, 6 - rod

The second step is to adjust the loading angle. According to the design of the experimental bench (Fig. 2), the loading of the model of the cutting wedge of the equal cutting force was carried out in a vertical plane. Adjustment of the position angle of the equal cutting force was carried out by orienting the fastening plate 2 with the model of the cutting wedge 4 installed on it in the frame 1 of the stand. The angle of the resultant cutting force was fixed by means of bolts. The values of the installation angles were selected based on the analysis of the influence of the cutter geometric parameters on the components of the cutting force: $\varphi_{R}=50^{\circ}$ and $\varphi_{R}=62^{\circ}$ [2, p. 211].

Third stage: loading. The model was loaded at the polarization-projection installation PPU-7 (Fig. 1). During the experiment, the model of the cutting wedge was successively loaded and the load was removed from it. Loading was carried out using a screw from which the load was transmitted to the rod 6 . The calibration disk 5 was used as an active force indicator. The load was gradually carried out by a power jack and was strictly controlled in order of stripes in the center of the disk. The model was kept under load, and then photographed by a digital camera. 

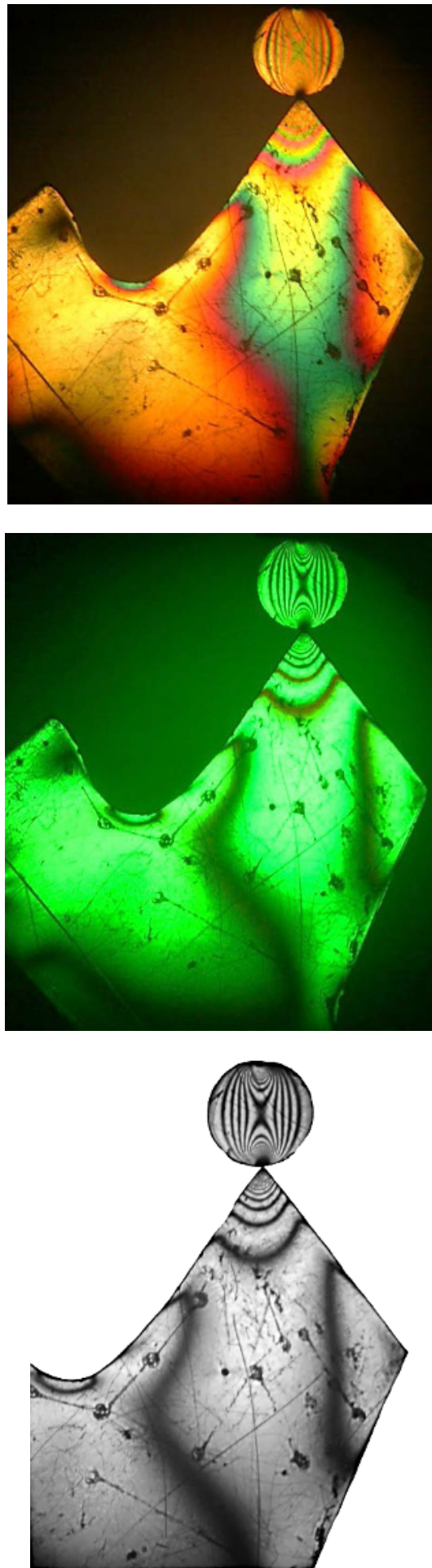

b
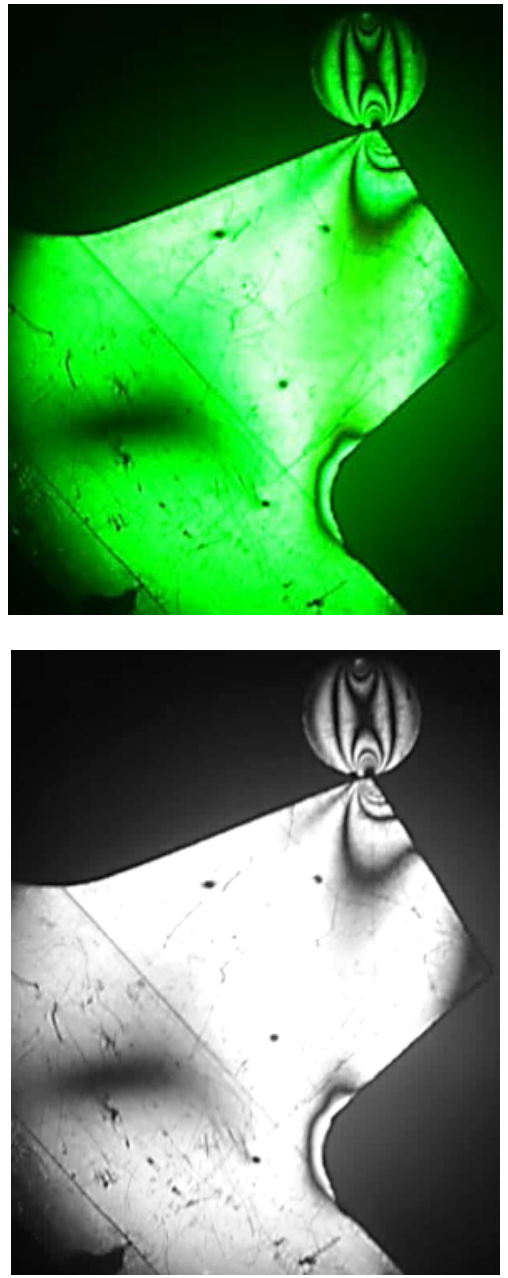

c

Fig. 3. Photograms of cutting wedge models with positive (left) and negative (right) face angle: $\mathbf{a}$ - in white light; $\mathbf{b}$ - in the light of a mercury lamp; $\mathbf{c}$ - in gray light 

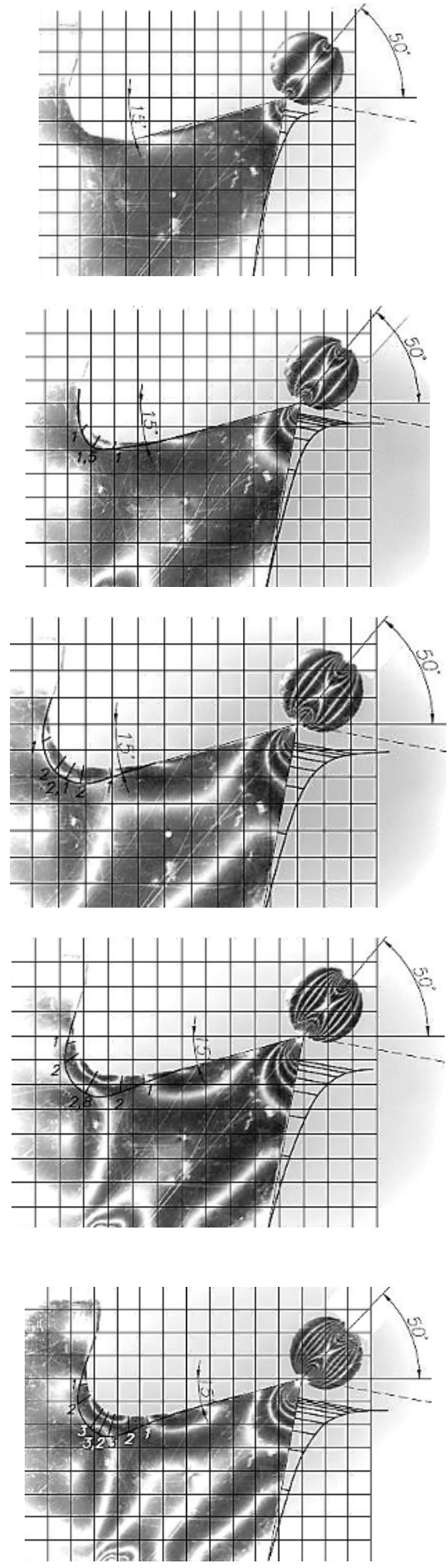
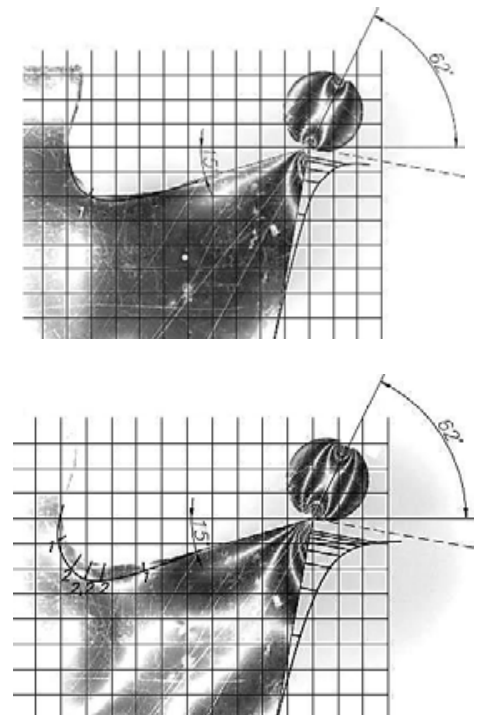

b

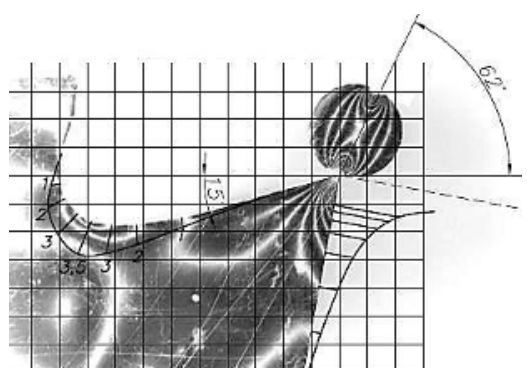

c

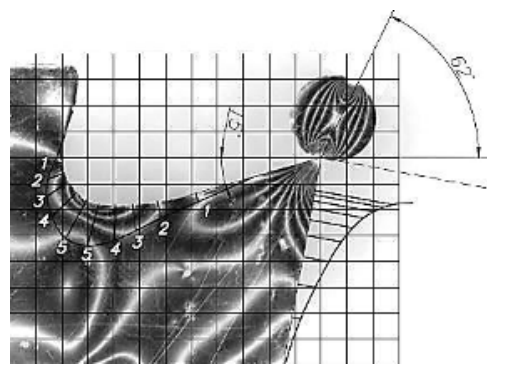

d

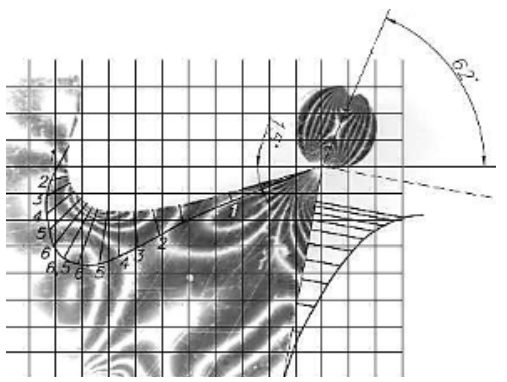

e

Fig. 4. The model of a cutting wedge with a positive face angle with a resultant force with an angle $\varphi_{R}=50^{\circ}$ (left) and $\varphi_{R}=62^{\circ}$ (right) and gradual loading (the force is expressed in the order of stripes): $\mathbf{a}-n=1, \mathbf{b}-n=2, \mathbf{c}-n=3, \mathbf{d}-n=4, \mathbf{e}-n=5$ 

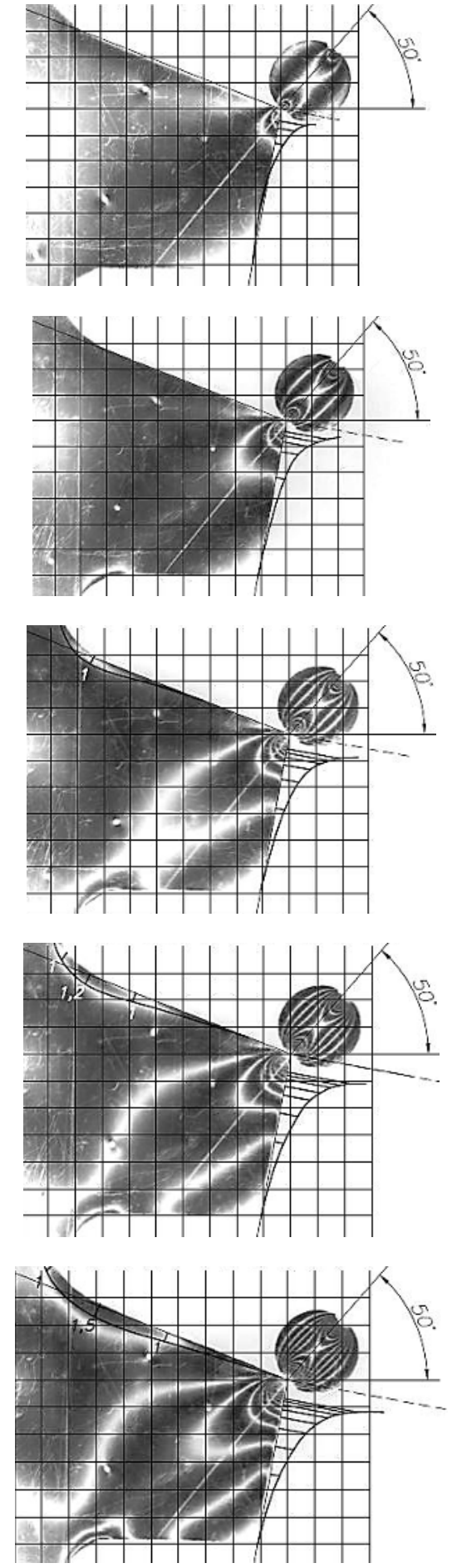

d

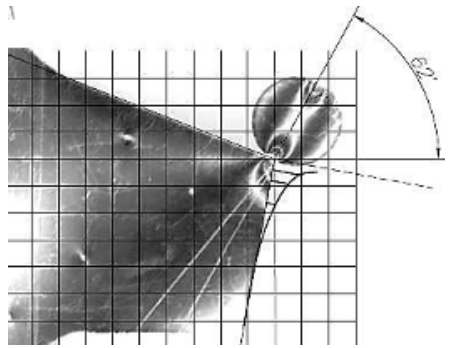

a

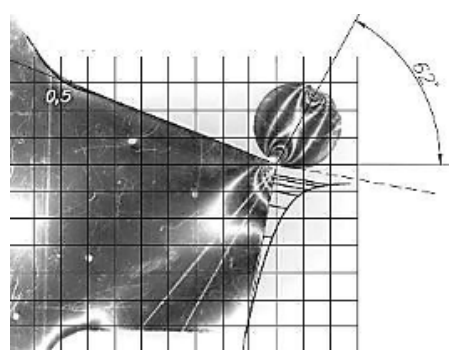

b
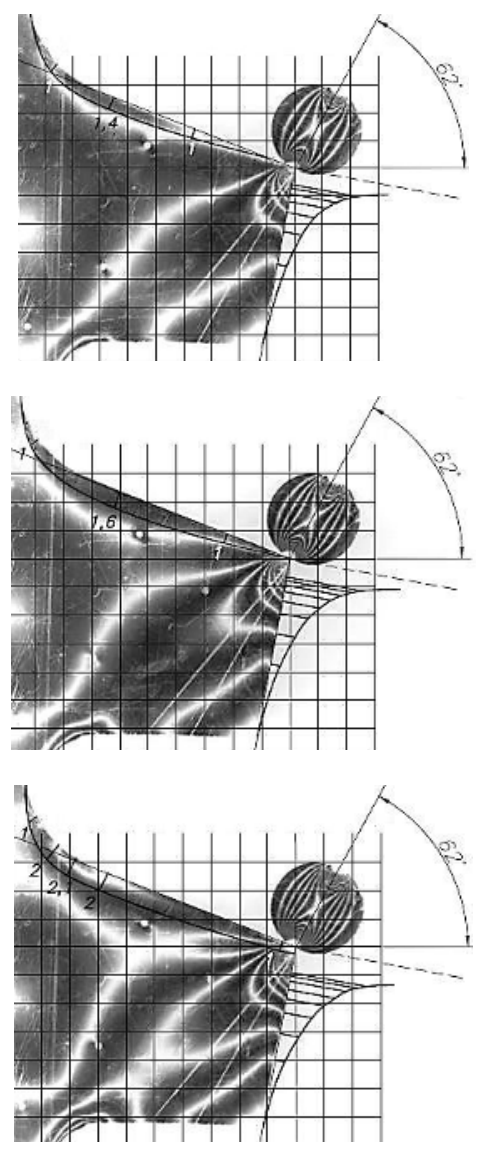

Fig. 5. The model of a cutting wedge with a negative face angle with a resultant force with an angle $\varphi_{R}=50^{\circ}$ (left) and $\varphi_{R}=62^{\circ}$ (right) and gradual loading (the force is expressed in the order of stripes): $\mathbf{a}-n=1, \mathbf{b}-n=2, \mathbf{c}-n=3, \mathbf{d}-n=4, \mathbf{e}-n=5$ 
The fourth stage: taking photograms. Photographing was carried out in the laboratory in the absence of background light. During the experiment, parts of optically sensitive transparent materials were transmitted by flat polarized light, as well as by light polarized in a circle. When examined in white light (polarized in a circle), patterns of colored stripes isochrome - were observed on the installation screen, which connected points having the same difference in main stresses (Fig. 3, a). When examined in the light of a mercury lamp (flat polarized light), dark lines were superimposed on the color image of the model isoclines connecting points with the same directions of main stresses (Fig. 3, b). The resulting isocline field characterized the direction of maximum stresses at model points.

The capture of experimental optical information was carried out by the digital camera, then experimental photograms were processed in Adobe Photoshop, where contrast and sharpness increased (Fig. 3, c), and then contour stresses epures were plotted in the AutoCAD program. Photograms of the stressed-strain state were obtained in the experiment, depending on the installation angle for two versions of the cutting wedge contour. Figure 3 shows examples of photograms for experimental variants: positive face angle $\gamma=+15^{\circ}$, angle of resultant cutting force $\varphi_{R}=62^{\circ}$; negative face angle $\gamma=-15^{\circ}$, angle of resultant cutting force $\varphi_{R}=50^{\circ}$. Epures of maximum stresses were plotted on the basis of the obtained photograms.

\section{Results and discussions}

Maximum stresses were determined by the standard algorithm of the photomechanics method $[1,3]$. The applied force was determined by the formula (1) according to the value of the order of strips in the center of the disk $n_{c}$ taking into account the optical constant $\sigma_{0}^{1,0}$ of the model material and initially for $n_{c}=1$ was $P=75 \mathrm{~N}$.

The value of the material strip of the models was determined taking into account the dependence:

$$
\sigma_{0}^{t}=\left(8 P_{i}\right) /\left(\pi \cdot d \cdot t \cdot n_{c}\right),
$$

where the thickness of the model $t=0.5 \mathrm{~cm}$. The value of the strip equaled $\sigma_{0}^{t}=3.9 \mathrm{MPa}$.

The maximum stresses on the photograms of the cutting wedge models according to the applied force, expressed by the order of the strips, respectively, were:

$$
\sigma_{\max }=\sigma_{0 i}^{t} \cdot n_{\max }=3.9 \cdot n_{\max }(\mathrm{MPa}) \text {. }
$$

The results of the graphoanalytic processing of the obtained photograms are given in Fig. 4 and 5, the final calculations of the maximum stresses are given in Fig. 6, a.

The analysis of the obtained photograms and the contour stresses of the cutting wedge models constructed by epures makes it possible to assert that a uniform stress compression state occurs along the contour of the rear surface for all versions of the geometry and orientation of the resultant cutting force.

Stress state of compression is observed on front surface in zone of application of resultant cutting force at insignificant section at tip of cutting wedge, value of which depends on loading level. And then on the front surface there is a zero point, which indicates a change in the stress sign.

After the zero point, a zone of tensile stresses appears on the front surface of the cutting wedge, which reduces its strength. The experiment investigated the influence of two factors: the magnitude of the face angle and the angle of application of resultant force. As a result of the analysis, it was found that the value of tensile stresses primarily depends on the geometry of the face angle. For positive face angles, the level of maximum tensile stresses on the front surface exceeds more than twice the level of similar stresses for a wedge with a negative face angle. 


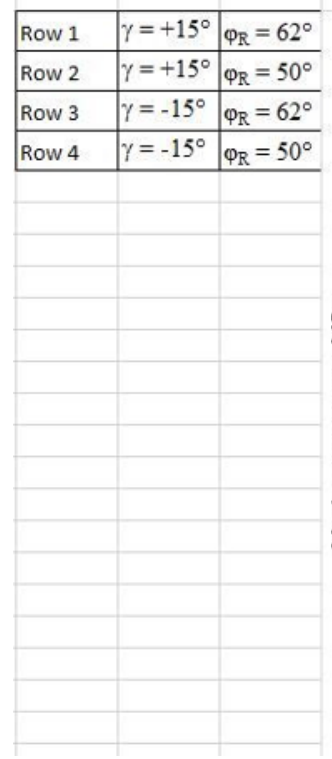

\section{Dependence of maximum stresses on loading conditions}

$\rightarrow$ Row $1 \rightarrow$ Row $2 \rightarrow$ Row $3 \rightarrow$ Row 4

30

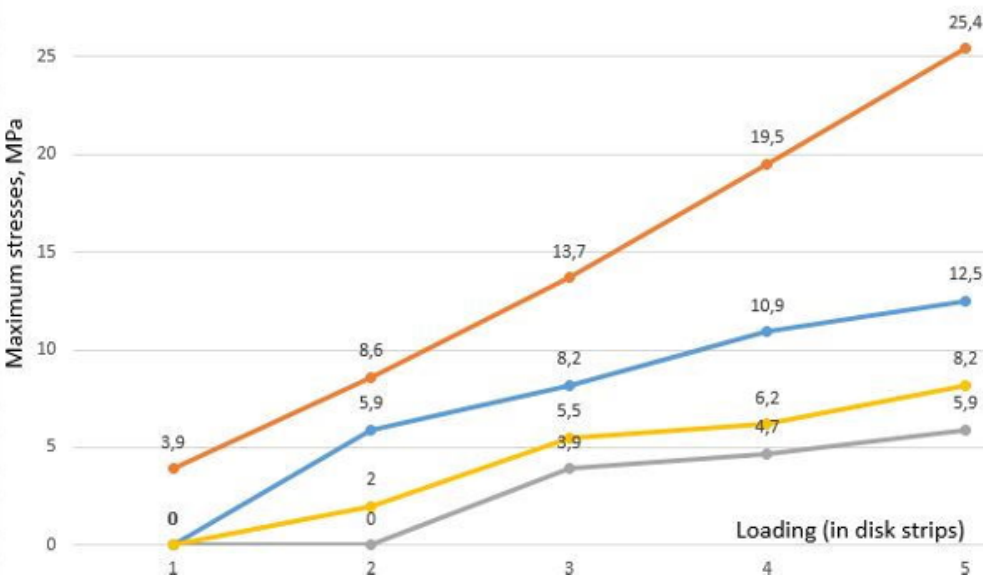

Dependence of maximum stresses on loading conditions

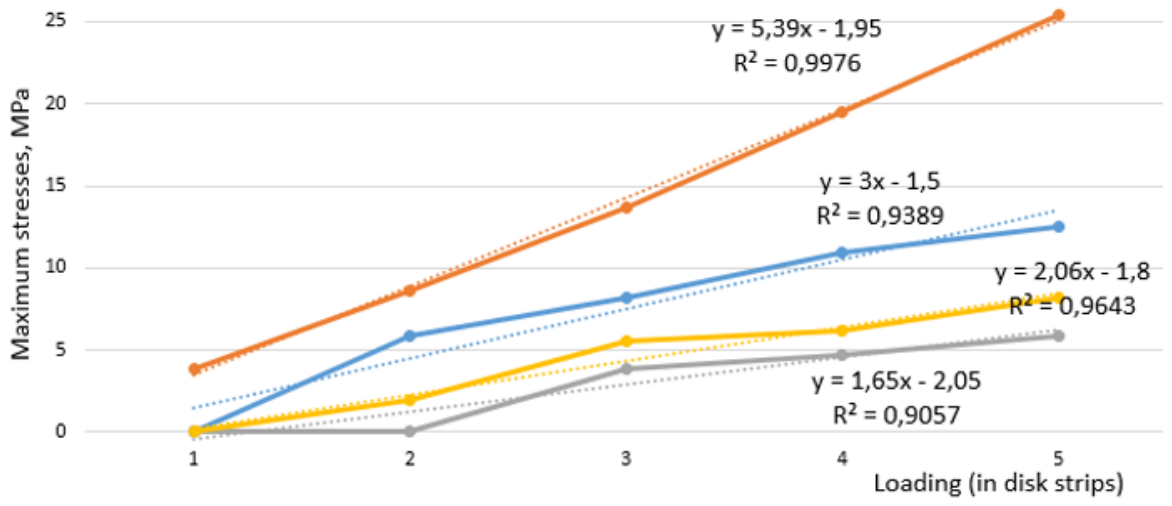

b

Fig. 6. Dependence of maximum stresses on loading conditions: a - results of analytical and graphical processing of photograms; $\mathbf{b}$ - trend lines

The trend lines given in Fig. 6 b show a good linear approximation of experimental data (for all dependencies, the determination coefficient $R^{2}>0.9$ ). The maximum intensity of formation of the stretching tension was detected for a positive face angle with an angle of application of the resultant cutting force $\varphi_{R}=62^{\circ}$. The minimum intensity of formation of the stretching tension was observed for a negative face angle with an angle of application of the resultant cutting force $\varphi_{R}=50^{\circ}$. For a positive face angle, it is also indicative that the effect of the resultant force application angle is much stronger than for a negative front angle.

Calculation-experimental information obtained by the method of photomechanics on models can be recommended in the practice of studying a cutting wedge and transferred to a real structure made of instrumental material taking into account geometric and power similarity. Stress field patterns can be used to create new mathematical models of tool strength. 


\section{References}

1. A.S. Koshelenko, G.G. Poznyak, Theoretical bases and practice of photomechanics in mechanical engineering, Izdatel'skiy dom "Granitsa", 296 p. (2004)

2. V.F. Bobrov, Fundamentals of the theory of metal cutting, Mechanical Engineering, 344 p. (1975)

3. O.V. Zhed, A.S. Koshelenko, G.G. Poznyak, V.A. Rogov, V.L. Fedorov, Mathematical and polarization-optical models of stresses in the cutting wedge, Bulletin of the Peoples' Friendship University of Russia. Series: Engineering Research, №1, pp. 21-26 (2000). 